\title{
Study of the Defibrillation Process in a Computer Model of Human Atria
}

\author{
Max Boegli ${ }^{1}$, Olivier Blanc ${ }^{1}$, Nathalie Virag ${ }^{2}$, Jean-Marc Vesin ${ }^{1}$, Lukas Kappenberger $^{3}$
}

\begin{abstract}
A realistic 3D anatomical model of human atria has been designed to study and better understand electrical propagation mechanisms. Besides normal sinus beat, pathological phenomena such as atrial flutter and fibrillation have been simulated. Having initiated atrial arrhythmias, our main objective is to test different defibrillation techniques by means of electrical shock application. Varying intensity and duration of electrical shocks, as well as the area of excited tissue, we were able to reproduce a curve of defibrillation success versus shock strength comparable to the ones obtained in human atria.
\end{abstract}

Key words - Computer modeling, arrhythmias, atrial fibrillation, defibrillation.

\section{INTRODUCTION}

Atrial fibrillation is the most frequent and costly arrhythmia, provoking heart failure and embolism. There is no biologic model to test therapeutic interventions, therefore there is a need to develop new research tools. Computer simulations offer the advantage of showing details difficult to study in nature and of being reproducible. Their major drawback is the heavy computational load needed for realistic modeling. Compared to the ventricles which are constituted of thick walls, atria have thin walls and therefore atrial arrhythmias can be considered as a two-dimensional problem in a 3D structure [1]. This allow us to simulate with current computer power a realistic model of atria taking into account the geometry.

The objective of this paper is to use this computer model of human atria to better understand and improve defibrillation techniques. Indeed, the mechanisms by which defibrillation shocks through stimulation electrodes situated distally from the myocardial tissue ("far-field" stimulation) can induce changes in transmembrane potentials is still an open question [2].

\section{METHODS}

The simulated atria are composed of two connected ellipsoids obtained by folding the 2D isotropic cardiac tissue described in [3] into a 3D model of one layer of cells. Orifices of appropriate size have been placed to simulate the veins (superior and inferior vena cava and the four pulmonary veins) and the valves (mitral and tricuspid valves). The cardiac tissue is composed of about $250^{\prime} 000$ cardiac cells and uses cellular membrane ion kinetics given by the Beeler-Reuter or Luo-Rudy models. Alternatively, a simplified but faster model using FitzHugh-Nagumo dynamics has been implemented.
To be able to build a model of the atria having a realistic size, we had to implement a monodomain model in order to keep a reasonable computational load. Therefore, the defibrillation shocks are applied by injecting transmembrane current to the cells located on surfaces parallel to the gradient of the applied electric field.
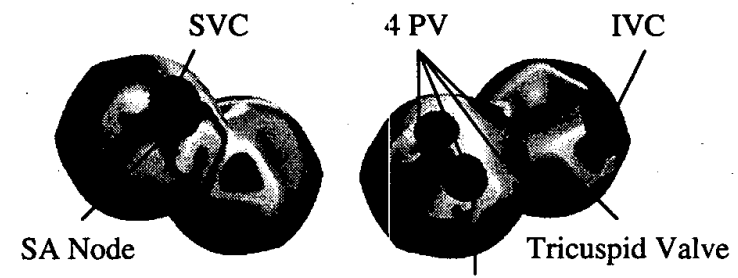

Mitral Valve

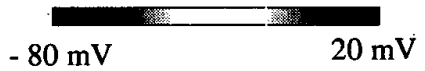

Figure 1: Snapshot of the cardiac electrical activity propagation in the virtual atria during atrial fibrillation. (Beeler-Reuter membrane ion kinetics).

\section{RESULTS}

Normal sinus beat was initiated from the sino-atrial (SA) node with a propagation speed of $50 \mathrm{~cm} / \mathrm{s}$. The addition of ectopic stimuli to the normal rhythm could induce sustain atrial arrhythmias: Atrial flutter displays as a periodic macroreentrant circuit, while atrial fibrillation can be seen as multiple reentering wavelets traveling randomly. These simulated arrhythmias show properties consistent with clinical observations [5].

Given a sustained atrial fibrillation (see an example in Figure 1), we applied electrical shocks of various intensity (transmembrane currents) and duration. By doing this for several values of shock timing, we obtained the curve of defibrillation success versus shock strength shown in Figure 2. These results obtained from numerical simulations are comparable to the typical sigmoidal curve from experimental results on rabbit and canine hearts [6].

One significant observation is that the application of a defibrillation shock may initiate a clepolarisation wave or postshock echo beat spreading through the tissue that may reinitiate the arrhythmia. This phenomenon is predominant for success rate around $50 \%$ (in the middle of the sigmoidal curve), where about half of the successful defibrillation shocks generated post-shock echo beats. 


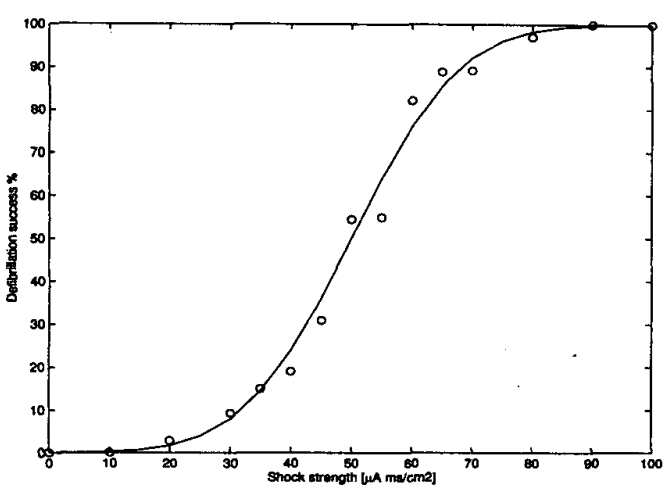

Figure 2: Defibrillation dose-response curve for the computer model of atria. The shock strength is computed as the transmembrane current density multiplied by the shock duration. The circles represent success rates obtained with the computer model (average over $n=20$ different sequences of atrial fibrillation). The plain line represents the experimental data on rabbit and canine hearts published in [4].

\section{CONCLUSIONS}

This model of human atria can reproduce clinical observations on atrial arrhythmias and it allowed us to test different defibrillation shocks strength and timing. The defibrillation dose-response curve obtained from simulations is comparable to the one obtained experimentally.

The advantage of this model is that it provides not only defibrillation success rates, but it also shows important phenomena such as post-shock echo beats that could reinitiate fibrillation. Therefore, this model can be used to study techniques improving the defibrillation process.

\section{REFERENCES}

[1] R.A Gray and J. Jalife, "Ventricular Fibrillation and Atrial Fibrillation are Two Different Beasts", Chaos, Vol. 8, No. 1, pp. 65-78, Mar. 1998.

[2] M.G. Fischler, "Syncytial Heterogeneity as a Mechanism Underlying Cardiac Far-Field Stimulation During Defibrillation-Level Shocks", Journal of Cardiovascular Electrophysiology, Vol. 9, No. 4, pp. 13101324, Apr. 1998.

[3] N. Virag, J.-M. Vesin, and L. Kappenberger, "A Computer Model of Cardiac Electrical Activity for the Simulation of Arrhythmias", PACE, Vol. 21 (Pt. II), No. 11, pp. 2366-2371, Nov. 1998.

[4] S.M. Dillon and K.F. Kwaku, "Progressive Depolarization: A Unified Hypothesis for Defibrillation Induction by Shocks", Journal of Cardiovascular Electrophysiology, Vol. 9, No. 5, pp. 529-552, May 1998.

[5] M.A. Allesie, P.L. Renama, J. Brugada, J.L.R.M. Smets, O. Penn, and C.J.H.J. Kirchhof, "Pathophysiology of Atrial Fibrillation", in Cardiac Electrophysiology From Cell to Bedside, pp. 548-559, Edited by D.P. Zipes and J. Jalife, W.B. Saunders Company, 1990.

\author{
1 Signal Processing Laboratory \\ Swiss Federal Institute of Technology \\ 1015 Lausanne - Switzerland \\ max.boegli@epfl.ch \\ olivier.blanc@epf.ch \\ jean-marc.vesin@epfl.ch \\ 2 Medtronic Europe SA \\ 1131 Tolochenaz - Switzerland \\ nathalie.virag@medtronic.com \\ ${ }^{3}$ Division of Cardiology, CHUV \\ 1011 Lausanne - Switzerland \\ lukas.kappenberger@chuv.hospvd.ch
}

